\title{
Análise de 10 anos de acidentes com material biológico entre a equipe de enfermagem*
}

Dayane Xavier de Barros ${ }^{1}$, Anaclara Ferreira Veiga Tipple ${ }^{2}$, Liwcy Keller de Oliveira Lopes Lima ${ }^{3}$, Adenícia Custódia Silva e Souza ${ }^{4}$, Zilah Cândida Pereira das Neves ${ }^{5}$, Thaís de Arvelos Salgado ${ }^{6}$

\author{
* Artigo extraído da dissertação de \\ Mestrado intitulada "Acidentes \\ ocupacionais com material biológico entre \\ a equipe de enfermagem no Estado de \\ Goiás" defendida na Faculdade de \\ Enfermagem da Universidade Federal de \\ Goiás, Goiânia, 2012. \\ ${ }^{1}$ Enfermeira, Mestre em Enfermagem. \\ Enfermeira da Secretaria de Estado de \\ Saúde do Distrito Federal. Docente do \\ curso de Graduação em Enfermagem pela \\ Escola Superior de Ciências da Saúde. \\ Brasília, DF, Brasil. E-mail: \\ dayanexavier@yahoo.com.br. \\ ${ }^{2}$ Enfermeira, Doutora em Enfermagem. \\ Professora Associada da Faculdade de \\ Enfermagem da Universidade Federal de \\ Goiás (UFG). Goiânia, GO, Brasil. E-mail: \\ anaclara@fen.ufg.br. \\ ${ }^{3}$ Enfermeira, Mestre em Enfermagem. \\ Docente do curso de Graduação em \\ Enfermagem da Faculdade de Ensino \\ Superior da Amazônia. Redenção, PA, \\ Brasil. E-mail: liwcykeller@yahoo.com.br. \\ ${ }^{4}$ Enfermeira, Doutora em Enfermagem. \\ Professora Voluntária do Programa de Pós- \\ Graduação em Enfermagem da UFG. \\ Professora Adjunto da Pontifícia \\ Universidade Católica de Goiás (PUC- \\ Goiás). Goiânia, GO, Brasil. E-mail: \\ adeniciafen@gmail.com. \\ ${ }^{5}$ Enfermeira, Doutora em Enfermagem. \\ Professora Assistente da PUC-Goiás. \\ Goiânia, GO, Brasil. E-mail: \\ zilahcandida@yahoo.com.br. \\ ${ }^{6}$ Enfermeira, Mestre em Enfermagem. \\ Discente do Programa de Pós-Graduação \\ em Enfermagem, nível Doutorado, da UFG. \\ Goiânia, GO, Brasil. E-mail: \\ thais.arvelos@hotmail.com.
}

Recebido: 01/12/2015.

Aceito: 14/05/2015.

Publicado: 30/06/2016.

Como citar esse artigo:

Barros DX, Tipple AFV, Lima LKOL, Souza ACS, Neves ZCP, Salgado TA. Análise de 10 anos de acidentes com material biológico entre a equipe de enfermagem. Rev. Eletr. Enf. [Internet]. 2016 [acesso em: ____18:e1157. Disponível em: http://dx.doi.org/10.5216/ree.v18.35493.

\section{RESUMO}

Os objetivos desse estudo foram: identificar o perfil dos acidentes com material biológico dos trabalhadores de enfermagem atendidos em um serviço de referência; caracterizar as condutas pré-exposição e analisar os fatores associados à exposição percutânea. Estudo epidemiológico, retrospectivo e analítico realizado em registros de acidentes envolvendo material biológico no período de 2000 a 2010 . O número de acidentes com a equipe de enfermagem foi de 2.569 representando $44,6 \%$ do total de registros. Predominaram exposições percutâneas envolvendo agulha com lúmen, sangue, entre técnicos de enfermagem do sexo feminino, nos membros superiores. Ser do sexo feminino e trabalhar fora do município onde se encontra o serviço aumentou cerca de duas vezes a chance de acidentes percutâneos. Os dados consolidam a importância do risco biológico no exercício da enfermagem e apontam para o fato de que os trabalhadores deslocam de seus municípios para o atendimento quando consideram o acidente grave, como os percutâneos.

Descritores: Exposição Ocupacional; Acidentes de Trabalho; Riscos Ocupacionais; Notificação de Acidentes de Trabalho; Equipe de Enfermagem.

\section{INTRODUÇÃO}

Os dispositivos perfurocortantes são instrumentos que frequentemente estão envolvidos nos acidentes ocupacionais entre os profissionais de enfermagem na prática laboral ${ }^{(1-2)}$. Dentre os microorganismos de maior relevância epidemiológica associados à exposição ocupacional, destacam-se os Vírus da Imunodeficiência Humana (HIV) e 
das Hepatites B (HBV) e C (HCV) ${ }^{(3)}$. Para o HIV, o risco de aquisição ocupacional é de um caso a cada 300 exposições percutâneas a sangue contaminado; para a Hepatite B, o risco varia de $6 \%$ a $30 \%$; e, no caso da Hepatite C é de $1,8 \%^{(3-4)}$.

Diversos estudos apontam a equipe de enfermagem como a que mais se expõe a material biológico e os dispositivos perfurocortantes os objetos mais frequentemente envolvidos nos acidentes ${ }^{(1-2)}$. Consequentemente, é a equipe que mais adquire infecções ${ }^{(5)}$. No Brasil, todos os cinco casos publicados de soroconversão ao HIV após exposição ocupacional ocorreu com membros da equipe de enfermagem ${ }^{(6-7)}$, o que reforça a interface do risco biológico e a equipe de enfermagem.

Para minimizar o risco de contaminação por micro-organismos infecciosos, há concordância entre as recomendações nacionais e internacionais sobre as medidas de segurança que devem ser implementadas antes e após a exposição. Entre as medidas pré-exposição, destaca-se a vacinação contra hepatite B e o uso correto dos equipamentos de proteção individual. São medidas pós-exposição os cuidados com o local do acidente, a utilização de imuno e quimioprofilaxia, quando indicadas, a notificação e o acompanhamento clínico-laboratorial $^{(3)}$.

Dado a relevância do tema, os acidentes ocupacionais envolvendo material biológico não podem ser considerados eventos cotidianos da prática profissional e devem ser permanentemente estudados com vistas a subsidiar medidas preventivas baseadas no conhecimento epidemiológico desse agravo. Levando em consideração as diferenças de condições de trabalho de cada contexto, não existem dados consolidados entre a equipe de enfermagem no período do estudo.

Os objetivos foram: identificar o perfil dos acidentes com material biológico entre profissionais de enfermagem atendidos em um serviço de referência; caracterizar as condutas pré-exposição nos registros de atendimentos de vítimas de acidentes com material biológico nesse grupo e analisar os fatores sociodemográficos e laborais associados à exposição ocupacional por material perfurocortante.

\section{MÉTODO}

Estudo epidemiológico, retrospectivo e analítico realizado em registros de acidentes com material biológico entre a equipe de enfermagem que buscou atendimento em um Hospital de Referência em Doenças Infectocontagiosas (HRDI) em um estado do centro- oeste brasileiro. Os dados foram coletados por meio do levantamento de prontuários de atendimentos dos profissionais da equipe de enfermagem, vítimas de acidentes com material biológico, após autorização prévia da instituição local do estudo e aprovação do comitê de ética em pesquisa da mesma instituição, sob o protocolo no $33 / 10$, atendendo as normas brasileiras para pesquisas com seres humanos.

A coleta de dados foi realizada no período de janeiro a julho de 2011, sendo utilizado um formulário semiestruturado, previamente avaliado e testado. Foram incluídos os registros de acidentes com profissionais da equipe de enfermagem no período do ano 2000 até os que o acompanhamento clínicolaboratorial foi concluído em dezembro de 2010 e excluídos os registros que não continham informações 
quanto ao "tipo de exposição" e "ano de acidente". Foi estabelecido como variável de desfecho o acidente percutâneo e as variáveis independentes foram sociodemográficas (sexo, idade e escolaridade) e aspectos laborais (categoria profissional, procedência do acidentado e status vacinal para hepatite B).

Os dados foram analisados no programa Statistical Package for Social Science (SPSS), versão 15.0 e foi utilizada estatística descritiva para caracterizar o perfil dos acidentes e as condutas pré-exposição e, também, análise univariada para estimar a chance (OR-odds ratio) de ocorrência de acidentes percutâneos, com intervalo de confiança de $95 \%$. As variáveis independentes que obtiveram valor de $p<0,10$ na análise univariada foram testadas em modelo de regressão logística multivariada forward likelyhood ratio.

\section{RESULTADOS}

Entre 2000 e 2010 o número de acidentes com a equipe de enfermagem foi de 2.569 representando $44,6 \%$ do total de registros. Desses, $10,1 \%$ eram de vítimas que sofreram mais de um acidente, podendo variar de dois até seis registros para o mesmo profissional. Em relação ao gênero, 91,6\% eram do sexo feminino e 8,4\% masculino. A mediana de idade foi de 33 anos, com mínima de 18 e máxima de 69 . Os técnicos de enfermagem foram os que mais sofreram acidente $(77,0 \%)$, seguido pelos auxiliares de enfermagem $(15,5 \%)$ e enfermeiros $(7,5 \%)$.

A Figura 1 apresenta a proporção de registros de acidentes com material biológico por categoria profissional segundo o ano de acidente.

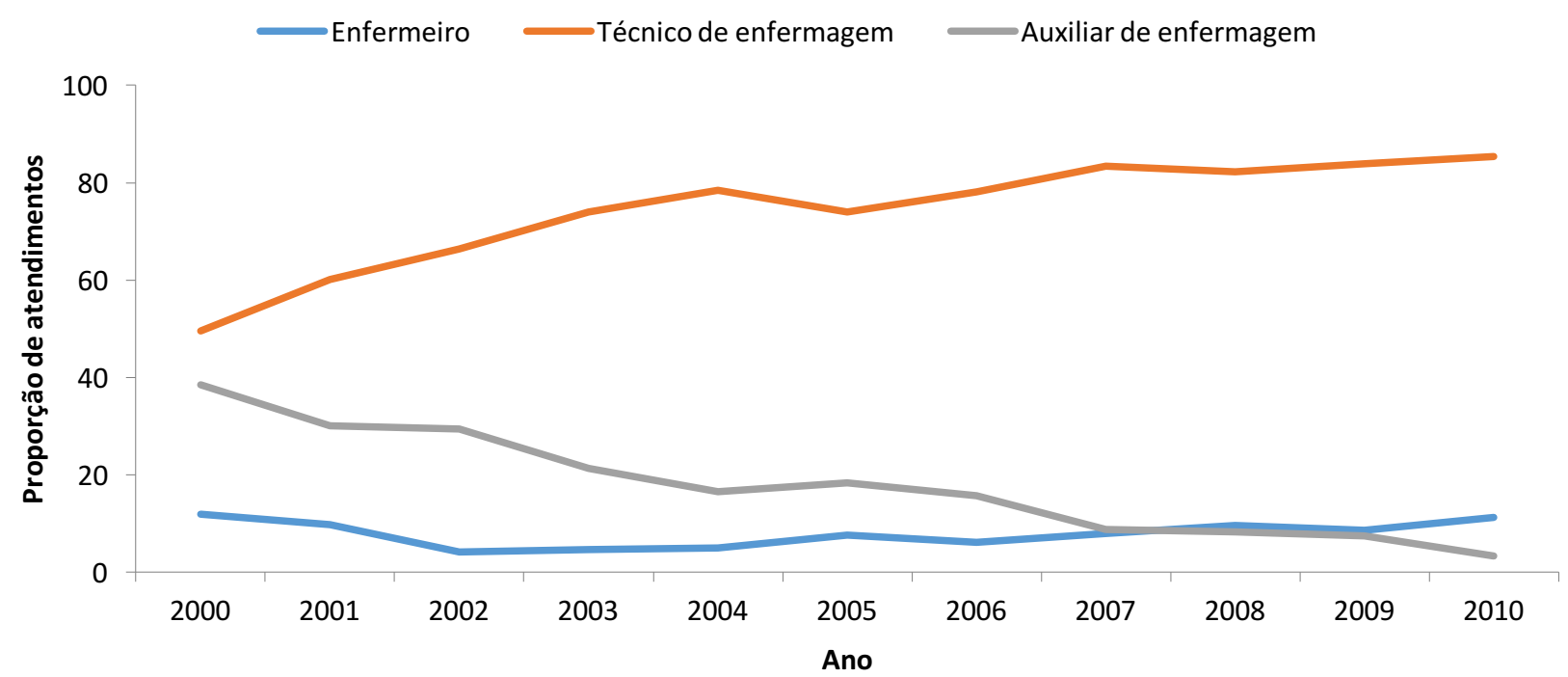

Figura 1: Proporção de acidentes com material biológico entre a equipe de enfermagem e ano de atendimento em um Hospital de Referência em Doença Infectocontagiosa no Estado de Goiás, de 2000 a 2010. Goiânia, GO, Brasil, 2012.

Observou-se que a proporção de atendimentos por acidente com os auxiliares de enfermagem apresentou queda ao longo dos anos $\left(x^{2}\right.$ de tendência $\left.=162,2 ; p=0,000\right)$ comparativamente aos atendimentos de técnicos de enfermagem e enfermeiros.

A maioria dos registros era de vítimas que trabalhavam em Goiânia (1.812/2.569; 70,5\%), 707 Rev. Eletr. Enf. [Internet]. 2016 [acesso em:______; 18:e1157. Disponível em: http://dx.doi.org/10.5216/ree.v18.35493. 
trabalhavam em outros municípios e em 52 não havia identificação da procedência. A Figura 2 mostra que a proporção de atendimentos de profissionais que trabalham em outros municípios aumentou com o tempo ( $x^{2}$ de tendência=137,7; $p=0,000$ ).

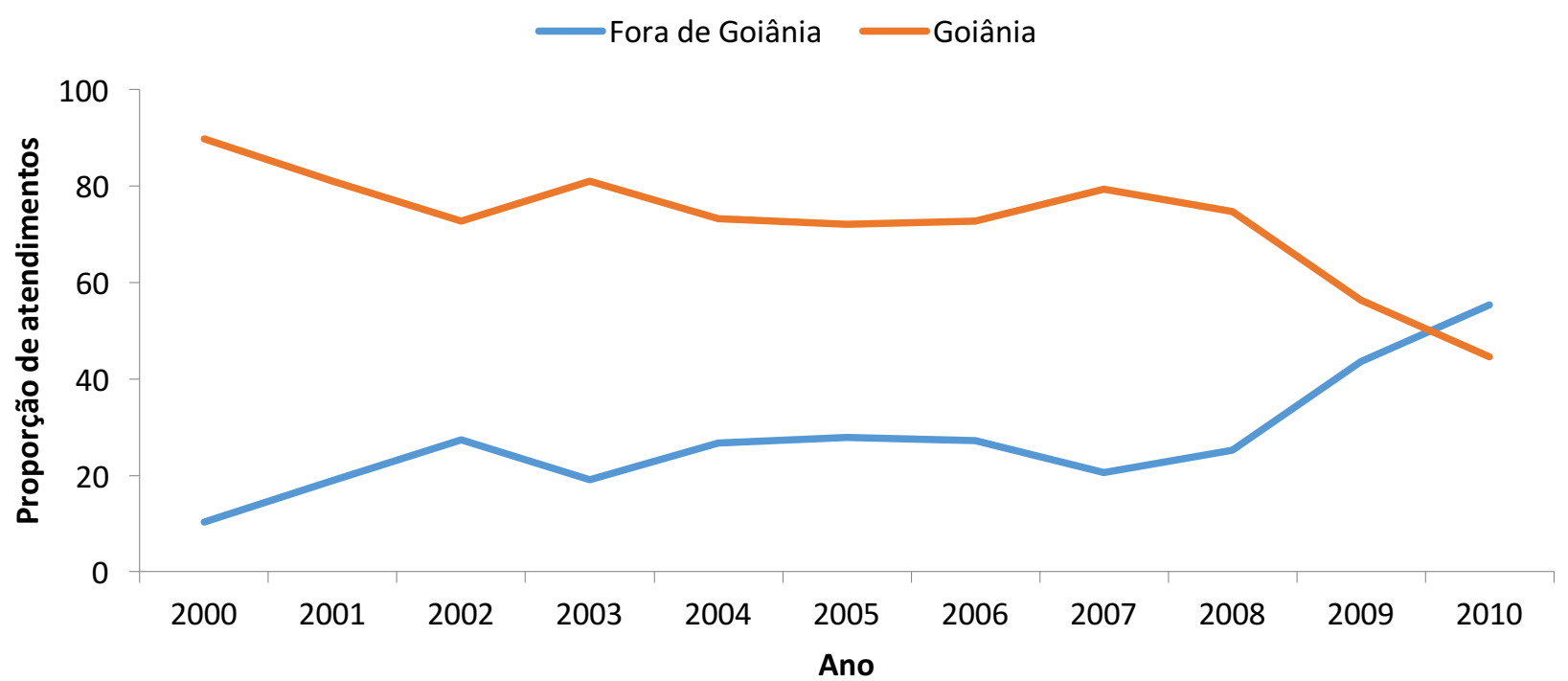

Figura 2: Distribuição da procedência de vítimas de acidente com material biológico em registro ( $N=2.569)$ de trabalhadores de enfermagem atendidos no Hospital de Referência em Doença Infectocontagiosa no Estado de Goiás, de 2000 a 2010. Goiânia, GO, Brasil, 2012.

Em $858(33,4 \%)$ registros não foi informado o tipo de instituição do acidentado. Para os casos informados, 60,2\% (1.030/1.711) eram oriundos de instituições privadas/conveniadas, 35,8\% de instituições públicas (612/1.711) e 4,0\% (69/1.711) filantrópicas. Em 63,8\% dos atendimentos as vítimas tinham procurado o serviço por conta própria, não havia qualquer documento de encaminhamento formal da instituição empregadora solicitando atendimento. Em 9,1\% dos casos havia o registro de preenchimento da Comunicação de Acidente de Trabalho.

A prevalência dos acidentes envolvendo materiais perfurocortantes foi de 89,5\% (2.299/2.569), desses, a agulha com lúmen foi o instrumento envolvido na maioria das exposições percutâneas $(1.775 / 2.299 ; 77,2 \%)$. As exposições em mucosa (oral/ocular), pele íntegra, pele não íntegra e mordedura obtiveram, respectivamente, $8,2 \%, 1,1 \%, 1,0 \%$ e $0,2 \%$ dos registros. A informação sobre a área corporal foi encontrada em $88,4 \%(2.271 / 2.569)$ dos registros, sendo que $86,8 \%(1.971 / 2.271)$ envolveram os membros superiores, 9,9\% (225/2.271) a face, 3,0\% (68/2.271) os membros inferiores e 0,3\% (7/2.271) o tronco. Em $75,2 \%$ (1.932/2.569) dos registros tinham informações sobre o material biológico envolvido nos acidentes e o sangue representou $88,6 \%(1.711 / 1.932)$ dos casos.

Em 45,5\% (1.169/2.569) dos registros não havia identificação das circunstâncias dos acidentes. Quando informadas (1.400), as principais frequências foram: manuseio de resíduos com 21,9\% (307/1.400), o que incluiu o descarte inadequado, manejo de caixa de perfurocortante e o reencape de agulha; o manejo de acesso vascular com 20,8\% (291/1.400) como a punção venosa/arterial, retirada de acesso venoso e a 
coleta de sangue; e em seguida, com 16,8\% (236/1.400) a administração de medicamentos. A punção digital foi descrita isoladamente como o procedimento de maior registro nas exposições, com 17,4\% (244/1.400).

Quanto à situação vacinal contra a hepatite B, 77,6\% (1993/2569) apresentavam esquema completo. Entre esses, 82 (4,1\%) havia referência quanto a realização do anti-HBs, sendo 59/82 (72,0\%) caracterizados como respondedores à vacina e $23 / 82(28,0 \%)$ não-respondedores. Os casos $(16,6 \%)$ com informação de vacinação incompleta foram incluídos no grupo de não vacinados. Em 4,3\% dos registros não havia essa informação e em 1,5\% estava registrado o desconhecimento do status vacinal.

Informações referentes ao uso de EPI no momento do acidente não constavam em $84,7 \%$ (2.177/2.569) dos registros e quanto aos que informaram (392), $118(30,1 \%)$ mencionaram a não utilização de qualquer equipamento de proteção. Quando houve registro do uso do EPI (274/392; 69,9\%), a maioria $(245 / 274 ; 89,4 \%)$ era referente às exposições percutâneas, sendo a luva de procedimento o mais citado $(77,4 \%)$, os demais citados foram: jaleco $(10,6 \%)$; máscara $(8,8 \%)$; óculos $(5,8 \%)$ e sapato fechado $(3,3 \%)$.

A Tabela 1 apresenta a análise univariada das variáveis independentes para a ocorrência de acidentes percutâneos. Houve associação estatisticamente significativa para o sexo $(p=0,000)$, trabalhar fora de Goiânia $(p=0,000)$, idade $(p=0,006)$ e vacinação contra hepatite $B(p=0,052)$.

Tabela 1: Análise univariada dos fatores associados à ocorrência de acidentes percutâneos entre profissionais de enfermagem atendidos no Hospital de Referência em Doenças Infectocontagiosas, no período de 2000 a 2010. Goiânia, GO, Brasil, 2012.

\begin{tabular}{|c|c|c|c|c|c|c|c|}
\hline \multirow{3}{*}{ Variáveis independentes } & \multicolumn{4}{|c|}{ Acidente percutâneo* } & \multirow{3}{*}{ OR } & \multirow{3}{*}{$95 \%$ IC } & \multirow{3}{*}{ Valor de $p$} \\
\hline & \multicolumn{2}{|c|}{ Sim } & \multicolumn{2}{|c|}{ Não } & & & \\
\hline & $\mathbf{N}$ & $\%$ & $\mathbf{N}$ & $\%$ & & & \\
\hline \multicolumn{8}{|l|}{ Sexo } \\
\hline Feminino & 2121 & 92,3 & 231 & 85,6 & 2,01 & $1,38-2,91$ & 0,000 \\
\hline Masculino & 178 & 7,7 & 39 & 14,4 & 1 & & \\
\hline \multicolumn{8}{|l|}{ Idade } \\
\hline$>=35$ anos & 1019 & 44,4 & 96 & 35,7 & 1,44 & $1,10-1,87$ & 0,006 \\
\hline$<35$ anos & 1274 & 55,6 & 173 & 64,3 & 1 & & \\
\hline \multicolumn{8}{|l|}{ Escolaridade: superior completo } \\
\hline Não & 2005 & 90,2 & 231 & 88,2 & 1,24 & $0,83-1,85$ & 0,291 \\
\hline Sim & 217 & 9,8 & 31 & 11,8 & 1 & & \\
\hline \multicolumn{8}{|l|}{ Categoria } \\
\hline Técnico de Enfermagem & 1756 & 91,1 & 221 & 91,7 & 0,92 & $0,57-1,49$ & 0,748 \\
\hline Auxiliar de Enfermagem & 371 & 68,3 & 29 & 59,2 & 1,48 & $0,80-2,70$ & 0,222 \\
\hline Enfermeiro & 172 & 8,9 & 20 & 8,3 & 1 & & \\
\hline \multicolumn{8}{|l|}{ Local de trabalho } \\
\hline Fora de Goiânia & 667 & 29,6 & 38 & 14,5 & 2,47 & $1,73-3,53$ & 0,000 \\
\hline Goiânia & 1588 & 70,4 & 224 & 85,5 & 1 & & \\
\hline \multicolumn{8}{|l|}{ Vacinação para hepatite B } \\
\hline Não & 371 & 17,1 & 56 & 22,0 & 0,73 & $0,53-1,00$ & 0,052 \\
\hline Sim & 1795 & 82,9 & 198 & 78,0 & 1 & & \\
\hline
\end{tabular}

A Tabela 2 apresenta a análise ajustada de regressão logística para identificação dos fatores independentemente associados ao acidente percutâneo. 
Tabela 2: Fatores independentemente associados aos acidentes percutâneos entre profissionais de enfermagem no período de 2000 a 2010. Goiânia, GO, Brasil, 2012.

\begin{tabular}{cccc}
\hline Variável & OR ajustado* & IC 95\% & VALOR DE $\mathbf{p}$ \\
\hline Sexo feminino & 1,94 & $1,31-2,88$ & 0,001 \\
Procedência fora de Goiânia & 2,51 & $1,73-3,64$ & 0,000 \\
\hline
\end{tabular}

* OR ajustado por faixa etária, escolaridade, categoria profissional e vacinação para hepatite $B$.

As mulheres apresentaram quase duas vezes mais chance de sofrerem acidentes percutâneos quando comparados aos homens. E, os atendimentos de acidentes com profissionais que trabalhavam fora de Goiânia tiveram 2,51 vezes mais chance de serem acidentes com material perfurocortante quando comparados aos que trabalham em Goiânia.

\section{DISCUSSÃO}

A prevalência de acidentes entre a equipe de enfermagem encontrada nesse estudo foi semelhante aos achados de estudos nacionais e internacionais ${ }^{(1-2,5,8)}$ e diferente de um estudo conduzido em Massachusetts/EUA e outro em Malaca/Malásia cujos resultados indicaram a maior frequência de acidentes entre a equipe médica ${ }^{(9-10)}$. A ordem decrescente de registro de acidentes observada para técnico de enfermagem $(77,0 \%)$, auxiliar $(15,5 \%)$ e enfermeiro $(7,5 \%)$ é semelhante ao encontrado na literatura ${ }^{(1-2,11)}$. Vale lembrar que, proporcionalmente, os técnicos de enfermagem representam a categoria com maior número de profissionais na sua equipe e, ainda, a equipe de enfermagem representa o maior número de profissionais na equipe de saúde.

A identificação de $10,1 \%$ dos registros com indivíduos que tiveram mais de um acidente foi menor que o encontrado em estudos internacionais ${ }^{(12-13)}$ que obtiveram, respectivamente, $51,0 \%$ e $80,1 \%$.

A maioria dos registros de acidentes entre o sexo feminino $(91,6 \%)$ reflete a trajetória histórica da enfermagem que é exercida predominantemente por mulheres. Vários estudos ${ }^{(1-2,11-16)}$ revelam a prevalência do sexo feminino tanto na ocorrência quanto na notificação de acidentes com material biológico entre a equipe de enfermagem.

A mediana de idade encontrada (33 anos) entre os acidentados é semelhante ao encontrado em estudo conduzido em Minas Gerais ${ }^{(11)}$. Em estudos que utilizaram faixa etária, predominaram entre 20 a 30 $\operatorname{anos}^{(1,8,16)}$ e entre 30 a 40 anos $^{(2,17)}$.

No que se refere à procedência das vítimas, constatou-se que mais de $1 / 4$ dos atendimentos ocorreram com indivíduos que se acidentaram fora do município onde se encontra o serviço de referência. O fato de o HRDI constituir o único serviço de referência para atendimento de acidentes com material biológico até o ano de 2006, após isso, com a criação das Unidades Sentinelas, (propostas como estratégia de municipalização e descentralização das ações em saúde com a finalidade de compartilhar responsabilidades entre os gestores), parece ter contribuído para a relativa queda de atendimentos para trabalhadores de instituições de Goiânia, levando a compreensão de que houve uma redistribuição dessas vítimas que passaram a ter a opção de buscar as Unidades Sentinelas. Entretanto, observou-se que não houve 
diminuição dos atendimentos de trabalhadores de outros municípios do estado de Goiás.

Importante ressaltar que o deslocamento da vítima para o atendimento em outros municípios aumenta o tempo entre o acidente e o primeiro atendimento. O que dificulta o início precoce profilaxia pósexposição e aumenta o risco de infecção do trabalhador ${ }^{(3)}$.

A maioria dos casos atendidos foi de trabalhadores oriundos de instituições privadas/conveniadas $(60,2 \%)$ e sem encaminhamento $(63,8 \%)$ sinalizando uma deficiência relacionada à gestão do atendimento ao profissional vítima de acidente com material biológico nas instituições e a subnotificação de acidentes de trabalho. Esses resultados apontam para falha no cumprimento da Norma Regulamentadora 32 (NR32) ${ }^{(18)}$ no que se refere à implantação de programa de atendimento ao trabalhador acidentado e a obrigatoriedade no preenchimento do Comunicado de Acidente de Trabalho - CAT, recomendado na mesma norma e que teve baixíssima frequência $(9,1 \%)$.

A NR 32 tem por finalidade estabelecer as diretrizes básicas para a implementação de medidas de proteção à segurança e à saúde dos trabalhadores dos serviços de saúde, bem como daqueles que exercem atividades de promoção e assistência à saúde em geral ${ }^{(18)}$. Um avanço importante dessa normativa foi a coresponsabilização dos empregadores sobre a saúde dos trabalhadores de saúde.

O perfil predominante dos acidentes percutâneos, envolvendo agulhas com lúmen em membros superiores e tendo o sangue como o material biológico mais descrito é semelhante ao encontrado em outros estudos $^{(1-2,11-14)}$. Acidentes com agulhas ocas merecem maior atenção, pois oferecem maior risco, pelo potencial de armazenar um volume maior de sangue ${ }^{(3)}$.

Quanto às circunstâncias dos acidentes, observou-se que parte deles poderiam ter sido evitados pela adoção de medidas de precaução padrão, como aqueles causados pelo reencape de agulhas e o descarte inadequado de perfurocortantes. Comportamentos que foram responsáveis por elevada frequência de acidentes em outros estudos $2(2,8,11,14)$.

Ainda relacionado ao incorreto manejo de resíduos em serviços de saúde, outro aspecto importante é que diversas investigações têm mostrado que as consequências desse manejo inadequado vão além do trabalhador que faz a segregação incorreta, alcançando profissionais que, pela natureza do trabalho, não entram em contato direto com o paciente, mas manuseiam os resíduos oriundos dessa assistência a exemplo da equipe do serviço de higiene e limpeza e dos coletores externos de resíduos de serviço de saúde ${ }^{(19)}$.

Seguida à NR32(18) , foi publicada a Portaria no $939^{(20)}$ relacionada à obrigatoriedade da utilização de dispositivos de segurança em materiais perfurocortantes nos serviços de saúde e sua completa implementação em um prazo de três anos. Após esse período, essa Portaria foi revogada pela de no 1.748 de agosto de $2011^{(21)}$, em que permite o uso dos dispositivos de acordo com a disponibilidade no mercado. Pode-se observar um retrocesso nos mecanismos legais de defesa à saúde do trabalhador no Brasil. Estudo conduzido no Reino Unido identificou redução de $86 \%$ nos acidentes percutâneos após a introdução desses dispositivos de segurança ${ }^{(22)}$.

A taxa de vacinação contra hepatite B encontrada $(77,6 \%)$ está entre aquelas identificadas na literatura 
com índices variando entre $56 \%$ a $93,9 \%^{(2,10-11,14,17)}$, revelando que o ideal de $100 \%$ ainda não foi alcançado, embora seja uma medida segura, gratuita e extremamente eficaz. Para o anti-HBs, o problema é ainda mais grave, pois o índice de registro de realização desse teste no nosso estudo foi baixo $(3,2 \%)$ e coincide com a literatura ${ }^{(17)}$. Na investigação realizada no hospital de referência para atendimento aos trabalhadores vítimas de acidente de trabalho com exposição a material biológico no município de Curitiba, a realização desse teste entre os atendimentos foi de $81,5 \%$, sendo que $13,9 \%$ foi não respondedor a vacina ${ }^{(1)}$.

A luva como EPI mais utilizado, foi citada em $77,4 \%$ dos registros em que essa informação estava presente e é semelhante ao encontrado na literatura ${ }^{(2,13)}$. As luvas não impedem a perfuração, mas funcionam como uma barreira mecânica que diminui o risco de contato com fluidos orgânicos, potenciais vinculadores de patógenos ${ }^{(22)}$.

Os achados desse estudo mostrando que ser do sexo feminino foi um importante fator predisponente para a ocorrência de acidentes percutâneos entre os profissionais da equipe de enfermagem, coincidem com outros autores ${ }^{(1,14)}$. Independentemente de haver um número menor de homens $(8,4 \%)$, as mulheres apresentaram aproximadamente duas vezes mais chance de sofrer um acidente percutâneo, podendo indicar uma maior procura por assistência quando da ocorrência desse tipo de acidente entre as mulheres, o que merece maior investigação.

Em relação à procedência das vítimas, os resultados permitem inferir que a notificação pode estar relacionada com o tipo de exposição. Entre as vítimas que trabalhavam fora de Goiânia as exposições mucocutâneas foram menos notificadas que as percutâneas, ou seja, os profissionais se deslocavam para notificar em outro município quando consideravam o acidente grave. Na literatura, a relação entre o tipo de acidente e sua notificação é evidenciada em estudos que concluíram que a taxa de notificação era maior quando o profissional considerava o acidente como de maior gravidade ${ }^{(23-24)}$

\section{CONCLUSÃO}

De 2000 a 2010 foram registrados 2.569 acidentes com membros da equipe de enfermagem em Hospital de Referência em Doenças Infectocontagiosas no estado de Goiás, perfazendo 44,6\% dos registros. Acidentes com perfurocortantes representaram cerca de 90,0\% e aconteceram, predominantemente, com agulhas com lúmen em membros superiores e na presença de sangue. A maioria das vítimas trabalhava em instituições particulares de Goiânia, não tinham sido formalmente encaminhadas e poucos registros continham informações sobre o preenchimento da CAT. A vacinação contra hepatite B foi a medida préexposição mais registrada e informações sobre o uso de equipamento de proteção estavam presentes em apenas $15,3 \%$ dos registros, sendo a luva o EPI mais relatado.

As variáveis sexo feminino e ser de fora do município de Goiânia, tiveram associação estatisticamente significativa e aumentaram em cerca de duas vezes a chance de ocorrência de acidentes percutâneos. Na maioria dos prontuários não havia registro de informações para diversas variáveis de investigação, o que impediu o aprofundamento da análise. O conhecimento detalhado das circunstâncias dos acidentes é 
importante para identificar as práticas desenvolvidas pelos profissionais que sugerem comportamentos de risco para a exposição a material biológico no ambiente laboral e fundamentar as medidas preventivas.

Nossos achados consolidam a importância do risco biológico no exercício profissional da enfermagem e sugerem que a implantação das unidades sentinela para o atendimento de vítimas com material biológico, ainda não apresentaram um resultado prático, pois os trabalhadores continuaram deslocando de seus municípios para o atendimento. E, provavelmente relacionada com essa dificuldade, os trabalhadores fazem uma avaliação do risco e buscam atendimento quando consideram o acidente grave, como os percutâneos.

A equipe de enfermagem precisa buscar mecanismos de enfrentamento do risco biológico. Protocolos de atendimento são necessários, locais especializados e equipes qualificadas também, entretanto a operacionalização dessas ações só acontecerá com trabalhadores conscientes do risco, dos seus direitos e das suas responsabilidades quanto à adesão às medidas pré e pós-exposição e ainda dos aspectos trabalhistas envolvidos. Cabe aqui destacar a responsabilização e comprometimento que deve haver do enfermeiro com a sua equipe na busca pela segurança no trabalho.

\section{Financiamento}

Essa pesquisa está vinculada à "Rede goiana de pesquisa em exposição de profissionais da área da saúde a material biológico", cadastrada junto à Fundação de Amparo à Pesquisa do Estado de Goiás (FAPEG) e recebeu financiamento por meio do edital 02/2007.

\section{REFERÊNCIAS}

1. Giancotti GM, Haeffner R, Solheid NLS, Miranda FMD, Sarquis LMM. Caracterização das vítimas e dos acidentes de trabalho com material biológico atendidas em um hospital público do Paraná, 2012. Epidemiol. Serv. Saúde [Internet]. 2014 [acesso em: 30 jun. 2016];23(2):337-46. Disponível em: http://dx.doi.org/10.5123/S1679-49742014000200015. 2. Jefferson Martins R, Saliba Moimaz SA, Isper Garbin AJ, Vicente Gonçalves PR, Saliba Garbin CA. Prevalência de Accidentes Com Material Biológico em um Municipio do Noroestede São Paulo, Brasil, no Periodo de 2007 a 2011. Cienc Trab [Internet]. 2014 [acesso em: 30 jun. 2016];16(50):93-6. Disponível em: http://dx.doi.org/10.4067/S071824492014000200006.

3. Rapparini C, Lara LTR, Vitória MAV. Recomendações para atendimento e acompanhamento de exposição ocupacional a material biológico: HIV e Hepatites B e C [Internet]. Brasília: Ministério de Saúde; 2004 [acesso em: 30 jun. 2016]. Disponível em: http://www.aids.gov.br/sites/default/files/manual_acidentes_final_0.pdf.

4. Cardo DM, Culver DH, Ciesielski CA, Srivastava PU, Marcus R, Abiteboul D, et al. A Case-Control Study of HIV Seroconversion in Health Care Workers after Percutaneous Exposure. N Engl J Med [Internet]. 1997 [acesso em: 30 jun. 2016];337(21):1485-90. Disponível em: http://dx.doi.org/10.1056/NEJM199711203372101.

5. Ippolito G, Puro V, Heptonstall J, Jagger J, De Carli G, Petrosillo N. Occupational human immunodeficiency virus infection in health care workers: worldwide cases through September 1997. Clin Infect Dis [Internet]. 1999 [acesso em: 30 jun. 2016];28(2):365-83. Disponível em: http://dx.doi.org/10.1086/515101.

6. Rapparini C. Occupational HIV infection among health care workers exposed to blood and body fluids in Brazil. Am J Infect Control. 2006 [acesso em: 30 jun. 2016];34(4):237-40. Disponível em:

http://dx.doi.org/10.1016/j.ajic.2005.08.016.

7. Lucena NO, Pereira FR, Barros FS, Silva NB, Alexandre MAA, Castilho MC, et al. Infecção pelo HIV-1 após acidente ocupacional, no Estado do Amazonas: primeiro caso documentado. Rev Soc Bras Med Trop [Internet]. 2011 [acesso em: 30 jun. 2016];44(5):646-7. Disponível em: http://dx.doi.org/10.1590/\$0037-86822011000500027.

8. Hajjaji Darouiche M, Chaabouni T, Jmal Hammami K, Messadi Akrout F, Abdennadher M, Hammami A, et al. 
Occupational blood exposure among health care personnel and hospital trainees. Int J Occup Environ Med [Internet]. 2014 [acesso em: 30 jun. 2016];5(1):57-61. Disponível em:

http://www.theijoem.com/ijoem/index.php/ijoem/article/view/321/456.

9. Massachusetts Department of Public Health Occupational Health Surveillance Program. Sharps Injuries among Hospital Workers in Massachusetts, 2010: Findings from the Massachusetts Sharps Injury Surveillance System [Internet]. Boston: Massachusetts Department of Public Health; 2010 [acesso em: 30 jun. 2016]. Disponível em: http://www.mass.gov/eohhs/docs/dph/occupational-health/injuries/injuries-hospital-2010.pdf.

10. Bhardwaj A, Sivapathasundaram N, Yusof M, Minghat A, Swe K, Sinha N. The Prevalence of Accidental Needle Stick Injury and their Reporting among Healthcare Workers in Orthopaedic Wards in General Hospital Melaka, Malaysia. Malays Orthop J [Internet]. 2014 [acesso em: 30 jun. 2016];8(2):6-13. Disponível em:

http://www.ncbi.nlm.nih.gov/pmc/articles/PMC4181076/.

11. Julio RS, Filardi MBS, Marziale MHP. Acidentes de trabalho com material biológico ocorridos em municípios de Minas Gerais. Rev Bras Enferm [Internet]. 2014 [acesso em: 30 jun. 2016];67(1):119-26. Disponível em:

http://dx.doi.org/10.5935/0034-7167.20140016.

12. Zhang MX, Yu Y. A study of the psychological impact of sharps injuries on health care workers in China. Am J Infect Control J [Internet]. 2013 [acesso em: 30 jun. 2016];41(2):186-7. Disponível em:

http://dx.doi.org/10.1016/j.ajic.2012.02.023.

13. Muralidhar S, Singh PK, Jain RK, Malhotra M, Bala M. Needle stick injuries among health care workers in a tertiary care hospital of India. Indian J Med Res [Internet]. 2010 [acesso em: 30 jun. 2016];131(3):405-10. Disponível em: http://www.ijmr.org.in/downloadpdf.asp?issn=0971-

5916; year=2010; volume=131;issue=3; spage=405; epage=410; aulast=Muralidhar; type=2.

14. Markovic-Denic L, Maksimovic N, Marusic V, Vucicevic J, Ostric I, Djuric D. Occupational exposure to blood and body fluids among health-care workers in Serbia. Med Princ Pract [Internet]. 2015 [acesso em: 30 jun. 2016];24(1):3641. Disponível em: http://dx.doi.org/10.1159/000368234.

15. Wu HC, Ho JJ, Lin MH, Chen CJ, Guo YL, Shiao JS. Incidence of percutaneous injury in Taiwan healthcare workers. Epidemiol Infect [Internet]. 2015 [acesso em: 30 jun. 2016];143(15):3308-15. Disponível em:

http://dx.doi.org/10.1017/s0950268815000321.

16. Beyera GK, Beyen TK. Epidemiology of exposure to HIV/AIDS risky conditions in healthcare settings: the case of health facilities in Gondar City, North West Ethiopia. BMC Public Health [Internet]. 2014 [acesso em: 30 jun.

2016];14(1):1283. Disponível em: http://dx.doi.org/10.1186/1471-2458-14-1283.

17. Jardim EMA, Carvalho PAM, Silva RP, Souza AC. Vacinação contra Hepatite B e resposta vacinal em trabalhadores da área da saúde envolvidos em acidentes com material biológico. Acta de Ciências e Saúde [Internet]. 2014 [acesso em: 30 jun. 2016];2(2):14-24. Disponível em: http://www.Is.edu.br/actacs/index.php/ACTA/article/view/58.

18. Portaria no 485, de 11 de novembro de 2005 (BR). Aprova a Norma Regulamentadora no 32 (Segurança e Saúde no Trabalho em Estabelecimentos de Saúde). Diário Oficial da União [Internet]. 16 nov. 2005 [[acesso em: 30 jun. 2016]. Disponível em: http://pesquisa.in.gov.br/imprensa/jsp/visualiza/index.jsp?jornal=1\&pagina=80\&data=16/11/2005.

19. Ream PSF, Tipple AFV, Barros DX, Souza ACS, Pereira MS. Biological risk among hospital housekeepers. Arch Environ Occup Health [Internet]. 2016 [acesso em: 30 jun. 2016];71(2):59-65. Disponível em:

http://dx.doi.org/10.1080/19338244.2014.927347.

20. Portaria no 939, de 18 de novembro de 2008 (BR). Diário Oficial da União [Internet]. 19 nov. 2008 [acesso em: 30 jun. 2016]. Disponível em:

http://pesquisa.in.gov.br/imprensa/jsp/visualiza/index.jsp?data=19/11/2008\&jornal=1\&pagina=238.

21. Portaria no 1.748, de 30 de agosto de 2011 (BR). Diário Oficial da União [Internet]. 31 ago. 2011 [acesso em: 30 jun. 2016]. Disponível em:

http://pesquisa.in.gov.br/imprensa/jsp/visualiza/index.jsp?data=31/08/2011\&jornal=1\&pagina=143.

22. Adams D, Elliott TS. Impact of safety needle devices on occupationally acquired needlestick injuries: a four-year prospective study. J Hosp Infect [Internet]. 2006 [acesso em: 30 jun. 2016];64(1):50-5. Disponível em: http://dx.doi.org/10.1016/j.jhin.2006.04.012.

23. Jagger J, Perry J, Parker G, Phillips EK. Nursing2011 survey results: Blood exposure risk during peripheral I.V. catheter insertion and removal. Nursing [Internet]. 2011 [acesso em: 30 jun. 2016];41(12):45-9. Disponível em: http://dx.doi.org/10.1097/01.NURSE.0000407678.81635.62. 
24. Machado-Carvalhais HP, Martins TC, Ramos-Jorge ML, Magela-Machado D, Paiva SM, Pordeus IA. Management of occupational bloodborne exposure in a dental teaching environment. J Dent Educ [Internet]. 2007 [acesso em: 30 jun. 2016];71(10):1348-55. Disponível em: http://www.jdentaled.org/content/71/10/1348.long. 